\title{
Inductance Aware Interconnect Scaling
}

\author{
Kaustav Banerjee \\ Center for Integrated Systems, Stanford University \\ Stanford CA 94305 \\ kaustav@ee.stanford.edu
}

\author{
Amit Mehrotra \\ Coordinated Science Lab, University of Illinois at Urbana-Champaign \\ Urbana IL 61801 \\ amehrotr@uiuc.edu
}

\begin{abstract}
This paper introduces a new global-tier interconnect scaling scheme which ensures that inductance effects do not start dominating the overall interconnect performance. It is shown that for unscaled global lines, inductance effects increase as technology scales while for the scaling scheme proposed by ITRS [1], interconnects become extremely resistive and, while inductance effects diminish with scaling but the performance, specifically, delay per unit length, degrades with scaling. The effect of the proposed global interconnect scaling scheme on optimized driver size, interconnect length, delay per unit length and total buffer area is quantified and compared with the unscaled and the ITRS cases. It is shown that the proposed scaling scheme improves the delay per unit length without degrading inductive effects or increasing buffer area with scaling.
\end{abstract}

\section{Introduction}

For deep submicron technologies using copper, on-chip inductive effects are a concern for signal integrity and overall chip performance [2]. Inductance effects in global interconnects are more severe due to the lower resistance per unit length of these lines which results in the reactive component of the interconnect impedance to become comparable to the resistive component, and also due to significant mutual inductive coupling between interconnects resulting from longer current return paths. With the recent adoption of Copper as the interconnect metal $[3,4]$, line resistances have decreased further and as a result, inductive effects have become more prominent. Hence inductance effects must be considered in the scaling schemes.

Accurate inductance modelling remains a challenging problem. This is due to the fact that magnetic fields have much longer spatial range compared to that of electric fields and therefore, in practical high-performance ICs containing several layers of densely packed interconnects the wire inductances are sensitive to even distant variations in the interconnect topology and switching activity. Moreover, uncertainties in the termination of neighbouring wires can significantly affect the signal return path and also the return current distributions, and therefore the effective loop inductance and resistance.

Present interconnect scaling schemes do not consider inductance. As it will be shown later, inductance effects start dominating with technology scaling when global lines are not scaled, while they start diminishing with scaling for ITRS where globaltier interconnect dimensions are scaled too aggressively (Table 1). The drawback of the ITRS approach is that the interconnect deProceedings of the International Symposium on Quality Electronic Design (ISQED'02)

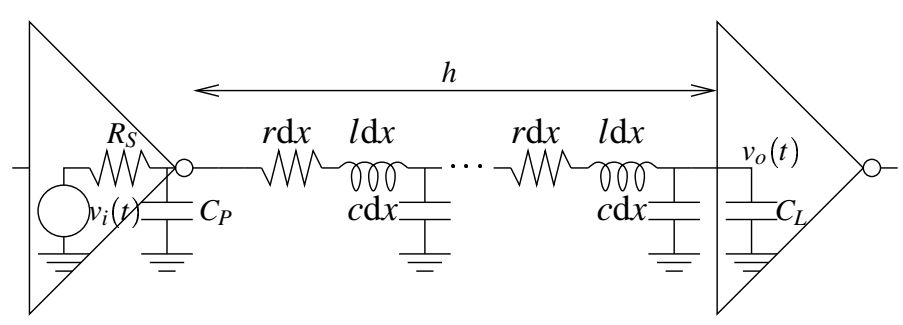

Figure 1: Equivalent circuit of a driver-interconnect-load segment. The interconnect is uniform with resistance, capacitance and inductance per unit length of $r, c$ and $l$ respectively.

lay increases since the line resistance per unit length dramatically increases with scaling.

This paper introduces a new scaling scenario where the global tier metal lines are scaled such that inductive effects remain approximately constant. The motivation behind such an approach is that current design tools and methodologies are able to satisfactorily cope with inductive effects in current technologies (180 nm and $130 \mathrm{~nm}$ ) but may not be able to do so if inductive effects increase with scaling. Finally, performance, area increase due to optimal buffering and wireability are compared for the three scaling scenarios.

\section{Preliminaries}

Consider a uniform line with resistance, capacitance and inductance per unit length of $r, c$ and $l$ respectively, driven by a repeater of series resistance $R_{S}$ and output parasitic capacitance $C_{P}$, and driving an identical repeater with load capacitance $C_{L}$ (Figure 1). For a given technology, let the output resistance, output parasitic capacitance and input capacitance of a minimum sized repeater be $r_{s}, c_{p}$ and $c_{0}$ respectively. Therefore if the repeater size is $k$ times the size of a minimum sized repeater, $R_{S}=r_{s} / k, C_{P}=c_{p} k$ and $C_{L}=c_{0} k$. Using the ABCD parameters of a uniform distributed $R L C$ line, It can be shown that the input-output transfer function can be approximated by a second order transfer function as discussed in [5].

$$
H(s)=\frac{V_{o}(s)}{V_{i}(s)}=\frac{1}{\left[1+s R_{S}\left(C_{P}+C_{L}\right)\right] \cosh (\theta)+\left[\frac{R_{S}}{Z_{0}}+s C_{L} Z_{0}+s^{2} R_{S} C_{P} C_{L} Z_{0}\right] \sinh (\theta)}
$$

where $Z_{0}=\sqrt{\frac{r+s l}{s c}}, \theta=\sqrt{(r+s l) s c h,} h$ is the length of the interconnect segment as shown in Figure 1 and $s$ is tl frequency $(j \omega)$. The step-response of this system $\mathrm{i}$

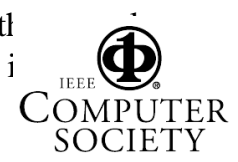




\begin{tabular}{|l||c|c|c|c|c|}
\hline Tech. node $(\mathrm{nm})$ & 180 & 130 & 100 & 70 & 50 \\
\hline width $(\mathrm{nm})$ & 525 & 382.5 & 280 & 195 & 137.5 \\
height $(\mathrm{nm})$ & 1155 & 1033 & 756 & 546 & 399 \\
$t_{\text {ins }}(\mathrm{nm})$ & 7699 & 6664 & 6022 & 5571 & 4116 \\
$\varepsilon_{r}$ & 3.75 & 3.1 & 1.9 & 1.5 & 1.25 \\
\hline$r(\mathrm{k} \Omega / \mathrm{m})$ & 36.3 & 60.1 & 103.9 & 206.6 & 401.3 \\
$c(\mathrm{pF} / \mathrm{m})$ & 269 & 240 & 154 & 125 & 106 \\
$l_{\max }(\mathrm{nH} / \mathrm{mm})$ & 9.2 & 10.9 & 13.5 & 17.9 & 18.8 \\
$h_{\text {opt }}(\mathrm{mm})$ & 3.33 & 2.5 & 2.22 & 1.32 & 1.06 \\
$k_{\text {opt }}$ & 174 & 151 & 110 & 82 & 53 \\
$\tau_{\text {opt }}(\mathrm{ns})$ & 0.165 & 0.147 & 0.125 & 0.089 & 0.071 \\
$r_{S}(\mathrm{k} \Omega)$ & 8 & 9.5 & 10 & 15.8 & 12.5 \\
$c_{0}(\mathrm{fF})$ & 1.9 & 1.7 & 1.5 & 1.3 & 1.2 \\
$c_{p}(\mathrm{fF})$ & 4.8 & 3.5 & 2.5 & 1.5 & 0.75 \\
\hline
\end{tabular}

Table 1: Technology parameters for top layer metal for different technology nodes as per ITRS.

$V_{o}(s)=\frac{1}{s} H(s)$ in the Laplace domain. However, computing the response in the time-domain is analytically intractable. The above transfer function is therefore approximated by a second order Padé approximation as

$$
H(s) \approx \frac{1}{1+s b_{1}+s^{2} b_{2}}=\frac{1}{\left(1+\frac{s}{s_{1}}\right)\left(1+\frac{s}{s_{2}}\right)}
$$

where

$$
\begin{aligned}
b_{1}= & R_{S}\left(C_{P}+C_{L}\right)+\frac{r c h^{2}}{2 !}+R_{S} c h+C_{L} r h \\
b_{2}= & \frac{l c h^{2}}{2 !}+\frac{r^{2} c^{2} h^{4}}{4 !}+R_{S}\left(C_{P}+C_{L}\right) \frac{r h^{2}}{2 !}+\left(R_{S} c h+C_{L} r h\right) \frac{r c h^{2}}{3 !} \\
& +C_{L} l h+R_{S} C_{P} C_{L} r h
\end{aligned}
$$

The $50 \%$ delay $\tau$ is given by

$$
0.5-\frac{s_{2}}{s_{2}-s_{1}} \exp \left(s_{1} \tau\right)+\frac{s_{1}}{s_{2}-s_{1}} \exp \left(s_{2} \tau\right)=0
$$

This transfer function can be used to calculate the 50\% delay [5]. Long VLSI interconnects are typically broken up into buffered segment of equal lengths and driven by identical repeaters. For minimum total delay in these long interconnects, the delay per unit length in the optimally buffered segment should be minimized. The driver size $k$ and interconnect length $h$ can be numerically optimized to give minimum delay per unit length $[5,6]$.

The second order transfer function given by (1) and discussed in $[5,6]$ can be critically damped, overdamped and underdamped when $b_{1}^{2}-4 b_{2}$ is equal to, greater than, or less than zero respectively. The response of an overdamped system is very similar to an RC line whereas for an underdamped system, the behaviour is significantly different from an RC line, i.e., inductive effects are significant. Since $b_{1}$ and $b_{2}$ are functions of $h$ and $k$ and $b_{2}$ is a function of $l$, It has been shown [5] that for optimum $h$ and $k$ for a given line inductance, a value $l_{\text {crit }}$ can be obtained for which the system will be critically damped [5]. If line inductance is less than $l_{\text {crit }}$, the system will be overdamped where as if line inductance is greater than $l_{\text {crit }}$, the system will be underdamped.

$$
l_{\text {crit }}=\frac{\frac{b_{1}^{2}}{4}-\frac{r^{2} c^{2} h^{4}}{4 !}-R_{S}\left(C_{P}+C_{L}\right) \frac{r c h^{2}}{2 !}-\left(R_{S} c h+C_{L} r h\right) \frac{r c h^{2}}{3 !}-R_{S} C_{P} C_{L} r h}{\frac{c h^{2}}{2 !}+C_{L} h}
$$

Proceedings of the International Symposium on Quality Electronic Design (ISQED'02)

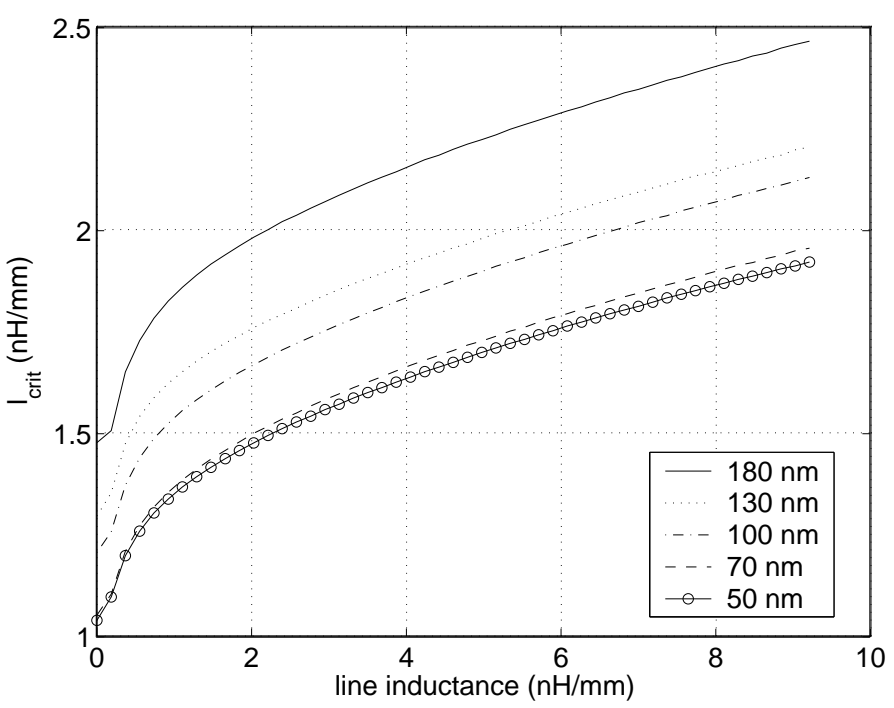

Figure 2: Critical inductance as a function of line inductance for various technology nodes with unscaled global lines.

\begin{tabular}{|c|c|c|}
\hline Tech. $(\mathrm{nm})$ & width $(\mathrm{nm})$ & aspect ratio \\
\hline \hline 180 & 525 & 2.2 \\
130 & 460 & 2.5 \\
100 & 425 & 2.7 \\
70 & 400 & 2.8 \\
50 & 380 & 2.9 \\
\hline
\end{tabular}

Table 2: Global tier line widths and aspect ratios for constant inductive effect scaling. Aspect ratios are the same as suggested by ITRS roadmap.

As pointed out earlier, the self, mutual and loop inductance of an interconnect is not just a function of geometry but also depends on the current return path which is a function of input vectors. Therefore, in this work we consider a conservatively large range of line inductances which include variations in the self, loop and coupling inductance of the interconnect.

Figure 2 plots the critical inductance as a function of $l$ for various technology nodes with unscaled global lines, while Figure 3 plots the critical inductance as a function of $l$ for various ITRS technology nodes with scaled global lines. Recall that the system is overdamped if $l<l_{\text {crit }}$. It can be observed from Figure 2 that the fraction of line inductance $l$ which is less than $l_{\text {crit }}$ decreases if global tier metal lines are not scaled with technology. On the other hand, Figure 3 shows that $l_{\text {crit }}$ increases with technology scaling for ITRS, i.e., the effect of inductance is diminishing with technology scaling. It can therefore be shown that [6] the range of line inductance $(l)$ values which are less than the corresponding $l_{\text {crit }}$ determine whether inductive effects are important for a given interconnect tier in a given technology.

\section{Proposed Scaling Scheme}

In this work, a new scaling scheme for global tier 1 ITRS technology nodes is proposed where inductive 


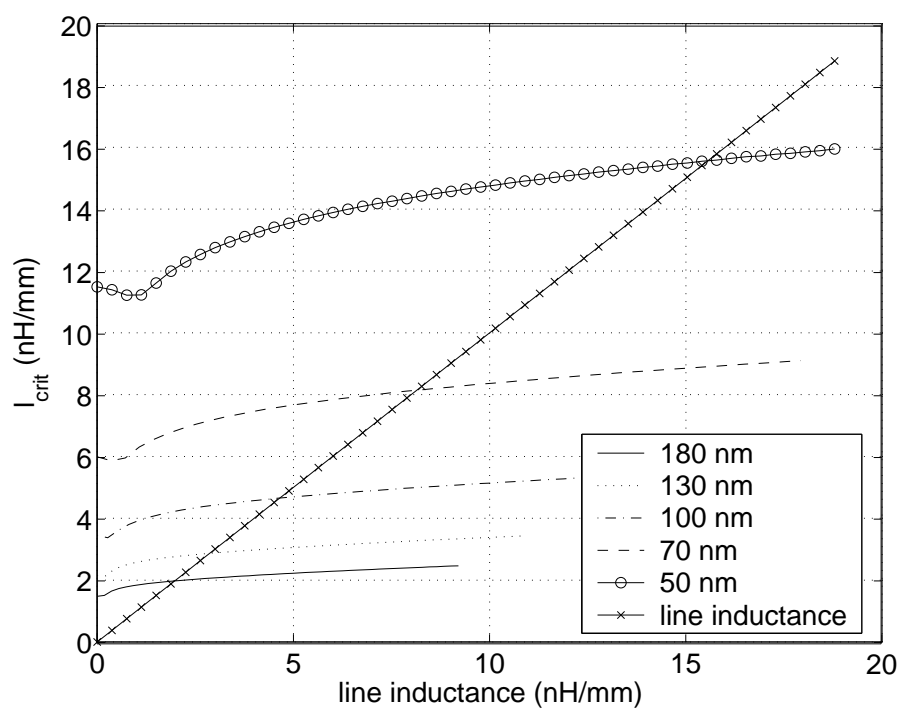

Figure 3: Critical inductance as a function of line inductance for various ITRS technology nodes.

\begin{tabular}{|c|c|c|c|c|}
\hline Tech. & $w$ & $\frac{\rho}{\rho_{0}}$ (thin-film) & $\frac{\rho}{\rho_{0}}$ (barrier) & {$\left[\frac{\rho}{\rho_{0}}\right]_{\text {eff }}$} \\
\hline \hline 180 & 525 & 1.0162 & 1.0487 & 1.0657 \\
130 & 382.5 & 1.0224 & 1.0663 & 1.0902 \\
100 & 280 & 1.0308 & 1.0914 & 1.1250 \\
70 & 195 & 1.0448 & 1.1351 & 1.1859 \\
50 & 137.5 & 1.0646 & 1.2003 & 1.2779 \\
\hline
\end{tabular}

Table 3: Resistivity ratios for the global tier metals for various ITRS technologies. All dimensions are in $\mathrm{nm}$. Barrier thickness of $10 \mathrm{~nm}$ assumed for all technology nodes.

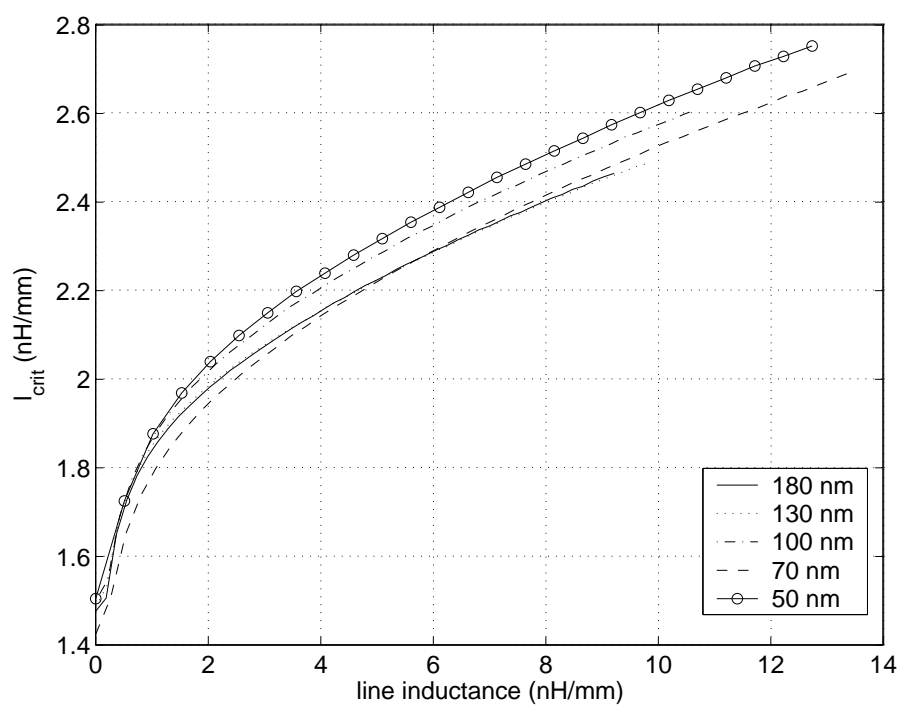

Figure 4: Critical inductance as a function of line inductance for various ITRS technology nodes with the proposed global tier interconnect scaling scheme. kept constant with scaling. The aspect ratio of global tier lines is same as that specified by the ITRS. Interconnect pitch is assumed to be twice the interconnect width for all technologies. For each technology, global tier line widths were varied until the $l_{\text {crit }}$ vs $l$ plot coincided with the $180 \mathrm{~nm}$ technology case. The proposed line widths and aspect ratios are shown in Table 2. Additionally, since the interconnect dimensions are comparable to the mean free path of electrons in $\mathrm{Cu}$, surface scattering starts having a non-negligible contribution to the resistivity compared to the contribution due to bulk scattering $[7,8]$. Therefore the resistivity of $\mathrm{Cu}$ interconnects needs to be appropriately increased. According to [7] the resistivity of a thin film of metal $\rho$ can be expressed in terms of bulk resistivity $\rho_{0}$ as

$$
\frac{\rho_{0}}{\rho}=1-\frac{3}{2 k}(1-p) \int_{1}^{\infty}\left(\frac{1}{x^{3}}-\frac{1}{x^{5}}\right) \frac{1-e^{-k x}}{1-p e^{-k x}} \mathrm{~d} x
$$

where $k=d / \lambda_{m f p}, d$ is the smallest dimension of the film (in our case, the width), $\lambda_{m f p}$ is the bulk mean free path of electrons and $p$ is fraction of electrons which are elastically reflected at the surface. For Copper, $p=0.47$ and $\lambda_{m f p}=421 \AA$ at $0^{\circ} \mathrm{C}$ [8]. Furthermore, since the thickness of the barrier material for Copper interconnects is not scaling with technology scaling, the effective area through which the current conduction takes place is reducing, therefore the effective resistivity of the interconnect is increasing [9]. The cumulative increase in resistivity of global tier lines due to these two effects for ITRS technology nodes is shown in Table 3. Similarly, the effective increase in resistivity for the metal line widths shown in Table 2 is also calculated. These modified resistivity values are used throughout the paper.

Figure 4 plots the critical inductance $\left(l_{c r i t}\right)$ for global tier lines for various technology nodes with the proposed scaling. It is clear that critical inductance values for all the technology nodes are almost same for a given value of line inductance over a very large range of line inductances. This implies that inductive effects are also very similar for all the technology nodes with the proposed scaling.

\section{Performance Comparisions}

We now compare optimized interconnect performance of the proposed scaling scheme for global wires with the ITRS and the unscaled cases for various technology nodes. Figure 5 shows the optimum interconnect length between two inverters $\left(h_{\text {opt }}\right)$ for minimum delay per unit length for the three cases for all technologies. It can be observed that $h_{\text {opt }}$ is largest for the unscaled case and smallest for the ITRS case. Also note that $h_{\text {opt }}$ decreases sharply with technology scaling for the ITRS case, whereas for the proposed scheme and the unscaled case, it does not change appreciably. Figure 6 shows the optimum driver size for the three cases for all technologies. Once again, a trend similar to the $h_{o p t}$ case is observed.

Figure 7 plots the optimized delay per unit length for the three cases for all technology nodes. The delay per unit length is the smallest for unscaled case and is the maximum for the ITRS case. It is instructive to note that for ITRS, the optimum interconnect delay per unit length increases with technology scaling. This is due to the fact the global tier interconnect dimensions are aggressively which dramatically increases line resistar '02) 


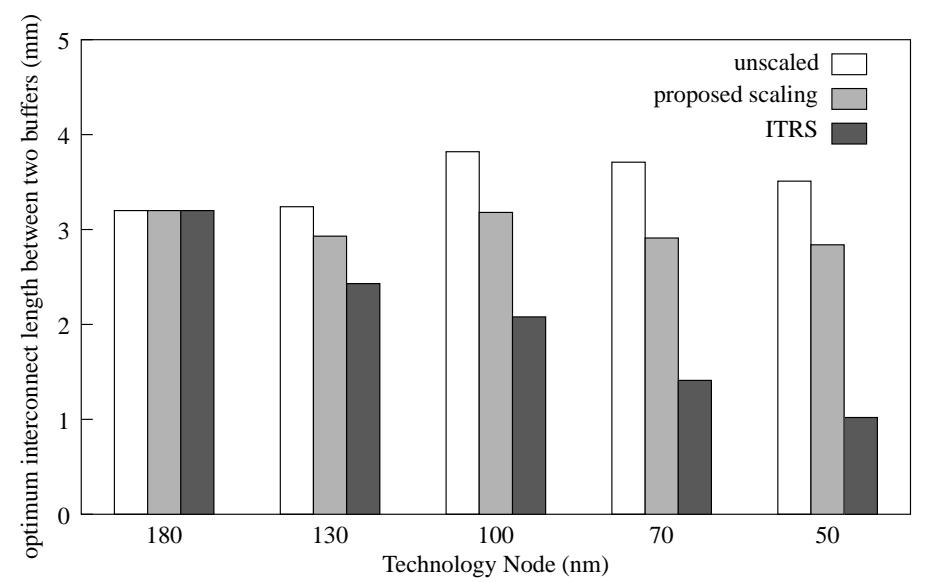

Figure 5: Optimum interconnect length between two buffers $\left(h_{\text {opt }}\right)$ for various technology nodes for global tier interconnects which are (a) unscaled, (b) scaled using the proposed scheme in Table 2 and (c) scaled according to ITRS specifications.

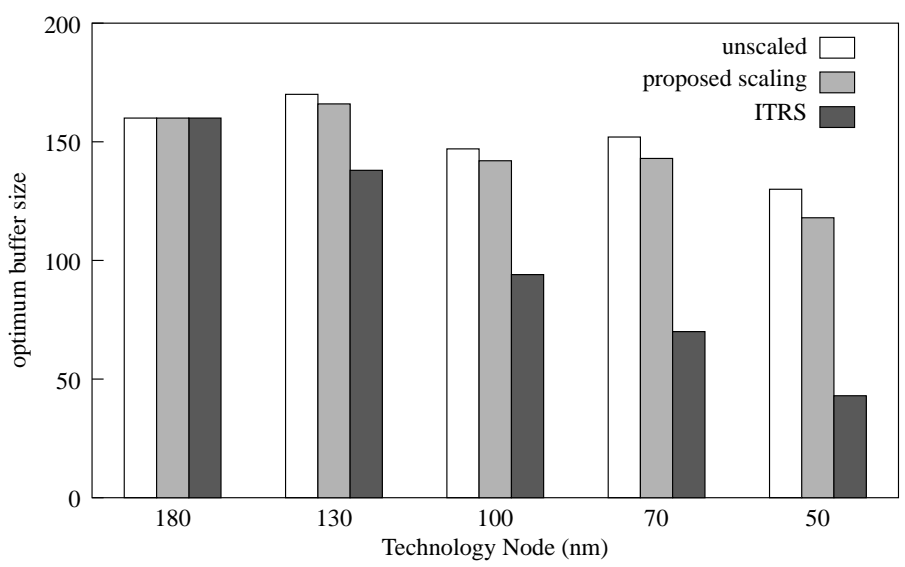

Figure 6: Optimum buffer size for various technology nodes for global tier interconnects which are (a) unscaled, (b) scaled using the proposed scheme in Table 2 and (c) scaled according to ITRS specifications.

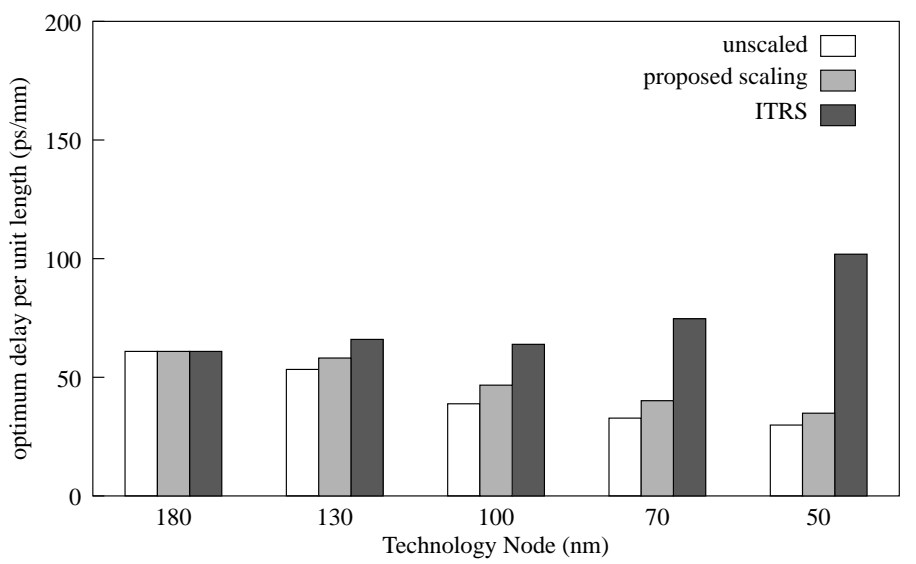

Figure 7: Optimum delay per unit length for various technology nodes for global tier interconnects, assuming a line inductance of $1 \mathrm{nH} / \mathrm{mm}$, which are (a) unscaled, (b) scaled using the proposed scheme in Table 2 and (c) scaled according to ITRS specifications.

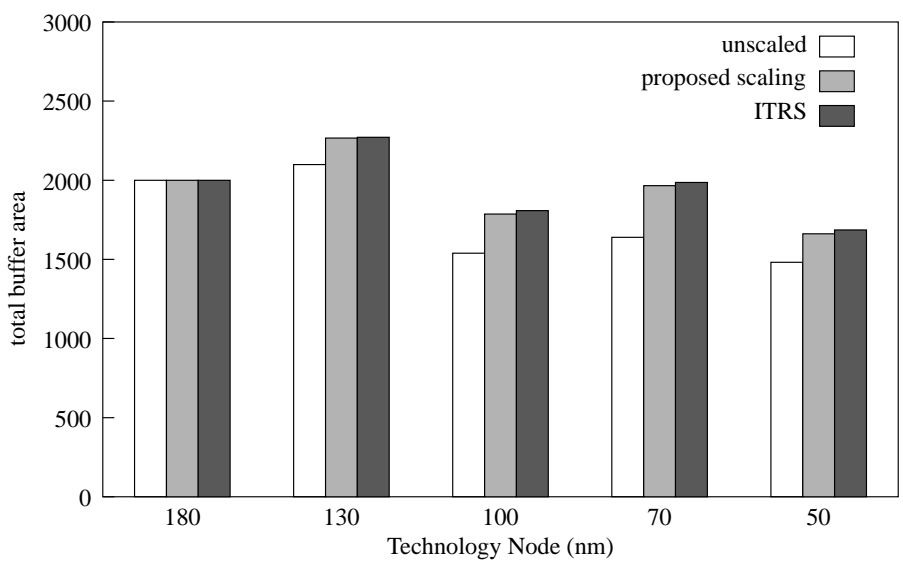

Figure 8: Total repeater area for an optimally buffered $4 \mathrm{~cm}$ global tier interconnect for various technology nodes which is (a) unscaled, (b) scaled using the proposed scheme in Table 2 and (c) scaled according to ITRS specifications. Repeater area is in terms of the area of the minimum sized inverter in the corresponding technology.

unscaled case and the proposed scaling of global wires, optimum interconnect delay per unit length decreases with technology scaling.

Note that while the optimum buffer size for ITRS reduces with scaling, $h_{\text {opt }}$ also reduces which increases the number of buffered segments for an interconnect of a given length. Figure 8 plots the total repeater area (i.e., number of repeaters $\times$ area of one repeater) in an optimally buffered $4 \mathrm{~cm}$ long global tier interconnect for all technologies. It can be observed that total repeater area is smallest for unscaled lines and largest for the ITRS lines. Additionally, the total repeater area in terms of the area of the minimum sized inverter in the corresponding technology does not change appreciably across technologies.

Finally, since the minimum wire width (pitch) for the global lines increases as a result of this scaling methodology, there may be some concern regarding the wireability of the chip creasing the number of metal layers or chip area. Ho creasi) 
cent analysis of chip wireability optimization has shown that the semiglobal and global tiers may not be packed to the maximum [9]. Consequently, higher tiers are actually routed within a larger than required area. Since the wire delay per unit length will decrease with increasing wire width it is also possible to re-route some of the global wires on the semi-global tier without violating the maximum allowable length (or delay) for that tier. Hence, the increased wire width resulting from this scaling methodology can be accommodated both in the global and the semiglobal tiers. Furthermore, this scaling scheme can be applied only to the performance critical paths of a chip where the susceptibility of performance to delay variations due to inductance effects is the highest. For non-critical paths, the ITRS based scheme may be used in order to save routing resouces.

\section{Conclusions}

In conclusion, a new global interconnect scaling scheme for deep sub-micron technology nodes is proposed which ensures that the inductive effects remain constant across technology nodes. It has been pointed out that for unscaled global lines, inductance effects increase as technology scales, while for the scaling scheme proposed by ITRS, interconnect becomes extremely resistive and inductance effects diminish with scaling but the performance, specifically delay per unit length, degrades with scaling. The effect of the proposed global interconnect scaling scheme on optimized driver size, interconnect length, delay per unit length and total buffer area is quantified and compared with unscaled and ITRS cases. It is shown that the proposed scaling scheme improves delay per unit length without changing inductive effects and buffer area consumption with scaling.

\section{References}

[1] "International technology roadmap for semiconductors (ITRS)," 1999.

[2] C.-K. Cheng, J. Lillis, S. Lin, and N. Chang, Interconnect Analysis and Synthesis. John Wiley \& Sons, 1999.

[3] D. Edelstein et al., "Full copper wiring in a sub-0.25 $\mu \mathrm{m}$ CMOS ULSI technology," in International Electron Devices Meeting. Digest of Technical Papers, pp. 773-776, 1997.

[4] N. Rohrer et al., "A 480MHz RISC microprocessor in a 0.12 $\mu \mathrm{m} \mathrm{L} \mathrm{L}_{\text {eff }} \mathrm{CMOS}$ technology with copper interconnects," in International Solid-State Circuits Conference. Digest of Technical Papers, pp. 240-241, 1998.

[5] K. Banerjee and A. Mehrotra, "Analysis of on-chip inductance effects using a novel performance optimization methodology for distributed RLC interconnects," in Proceedings 2001 Design Automation Conference, pp. 798-803, 2001.

[6] K. Banerjee and A. Mehrotra, "Accurate analysis of on-chip inductance effects and implications for optimal repeater insertion and technology scaling," in Proceedings 2001 IEEE Symposium on VLSI Circuits, pp. 195-198, 2001.

[7] J. C. Anderson, ed., The Use of Thin Films in Physical Investigations. Academic Press, 1966.

Proceedings of the International Symposium on Quality Electronic Design (ISQED'02) 0-7695-1561-4/02 \$17.00 @ 2002 IEEE

(1) 2002 IEEE
[8] F. Chen and D. Gardner, "Influence of line dimensions on the resistance of $\mathrm{Cu}$ interconnections," IEEE Electron Device Letters, vol. 19, pp. 508-510, Dec. 1998.

[9] K. Banerjee, S. J. Souri, P. Kapur, and K. C. Saraswat, "3-D ICs: A novel chip design for improving deep-submicrometer interconnect performance and system-on-chip integration," Proceedings of the IEEE, vol. 89, pp. 602-633, May 2001. 